
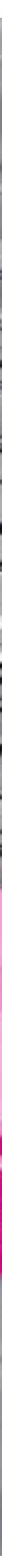

\title{
Propuesta innovadora para la introducción de la lectura en la escuela primaria
}

An Innovative proposal for the introduction of reading in elementary school Proposta inovadora para a introdução de leitura na escola primária 


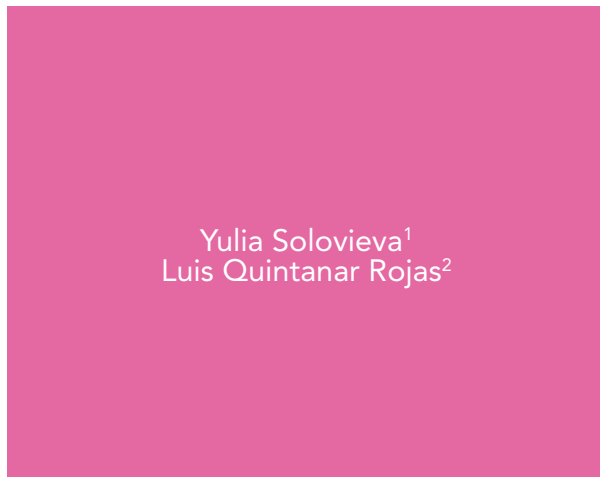

1. Ph.D. Coordinador y profesor - investigador de la Maestria en Diagnostico y Rehabilitación Neuropsicológica de la Facultad de Psicología de la BUAP (México); Doctor en ciencias Psicológicas por la Universidad Estatal de Moscú (Rusia) con especialización en Psicología del desarrollo y psicología pedagógica. Líneas de investigación: neuropsicología, rehabilitación neuropsicológica, psicología del desarrollo, psicología de la educación y métodos de enseñanza; correo electrónico: yulia.solovieva@corre.buap.mx

2. Ph.D.. Fundador y profesor - investigador de la Maestria en Diagnostico y Rehabilitación Neuropsicológica de la Facultad de Psicología de la BUAP (México); Doctor en ciencias Psicológicas por la Universidad Estatal de Moscú (Rusia) con especialización en la Neuropsicología. Líneas de investigación: neuropsicología, rehabilitación neuropsicológica, evaluación neuropsicológica d niños y adultos; correo electrónico: luis.quintanar@corre.buap.mx

Fecha de recepción: 24 de febrero de 2017 / fecha de aceptación: 24 de mayo de 2017

\section{Resumen}

El problema de la enseñanza de la lectura es de gran importancia para el sistema educativo. Este artículo presenta un método innovador para la introducción de la lectura, basado en el paradigma histórico-cultural y la teoría de la actividad. La lectura se concibe como actividad verbal que cumple diversas funciones en la vida, mientras que la enseñanza se da como formación de las acciones mentales por etapas en forma colectiva y guiada. El método permite evitar errores y favorecer los motivos cognitivos del niño.

Palabras clave: Enseñanza innovadora, métodos de enseñanza, método formativo, enseñanza de la lectura.

\section{Summary}

The problem of teaching reading is one of great importance to the educational system. This article shows an innovative method for the introduction of reading, based on the historical-cultural paradigm and the theory of activity. Reading is conceived as a verbal activity which fulfills various functions in life, while teaching is given as the formation of mental actions by stages in a collective and guided way. The method allows to avoid errors and to favor the cognitive motives of the child.

Key words: Innovative teaching, teaching methods, formative method, teaching of reading.

\section{Resumo}

O problema do ensino da leitura é de grande importância para o sistema de ensino. Este artigo apresenta um método inovador para a introdução de leitura, baseada na teoria de paradigma ea atividade histórico-cultural. Reading é visto como atividade verbal que tem muitas funções na vida, enquanto o ensino é dado como formação de ações mentais em estágios e guiado coletivamente. O método evita erros e promover razões cognitivo da criança.

Palavras chave: ensino inovador, métodos de ensino, métodos de ensino, o ensino da leitura. 
A veces, los que bablan de educación son lo que menos saben de ella.

Jean Baptiste Moliere Las preciosas ridículas

\section{Introducción}

El método empleado para la introducción inicial de la lectura, como unidad esencial del lenguaje escrito, parte de un problema importante en la escuela primaria, que no se ha solucionado con éxito debido a que los alumnos siguen con dificultades en la comprensión lectora y en la producción escrita a lo largo de todo el ciclo escolar. Al tiempo, los maestros continúan empleando métodos tradicionales sin modificarlos, algunos de ellos incluso de siglos pasados, pues también entonces hubo una versión oficial de la sabiduría y una crítica a la misma por parte de pensadores (ver epígrafe) insatisfechos con esa visión del mundo predominante.

La posibilidad de cambiar la situación del fracaso escolar se relaciona con la necesidad de crear métodos nuevos probados en la práctica. En tal sentido, Colombia cuenta con el Colegio Merani de Bogotá, el cual, denominado oficialmente como "experimental", tiene garantizada la oportunidad de probar nuevos planes de estudios y métodos de enseñanza, contando con un permiso oficial logrado gracias a los esfuerzos de su dirección.

En México no existe oficialmente el término de "colegio experimental" y ello no facilita la creación de nuevas alternativas. La preparación de los maestros está definida por los reglamentos de la Secretaría de Educación, lo cual limita las posibilidades de cambio y de reflexión sobre el beneficio de los programas y métodos existentes para el desarrollo de los alumnos. Las reformas educativas no consideran la preparación de los maestros, los planes de estudio, ni los métodos concretos de enseñanza, y la mayoría de programas de posgrado afines a la educación no muestran interés o capacidad para proponer algo alternativo; pareciera que el tema ni siquiera es importante para las autoridades o los especialistas en educación.

A pesar de ello, los autores del presente artículo han intentado modificar la situación, elaborando métodos innovadores a lo largo de 15 años en México. El escenario de dichos métodos se da, por un lado, en los trabajos de investigación realizados como tesis de la Maestría en Diagnóstico y Rehabilitación Neuropsicológica, de la Facultad de Psicología de la Universidad Autónoma de Puebla ${ }^{3}$, y por otro, en el colegio Kepler ${ }^{4}$ de la ciudad de Puebla; allí se efectuó un trabajo constante de modificación y creación de métodos de enseñanza innovadores, en conjunto con una capacitación de los maestros del nivel preescolar y primaria.

El presente artículo da cuenta del método innovador para la introducción de la lectura en el primer grado de la escuela primaria con niños mexicanos regulares; el cual se concentra en un trabajo experimental aplicado inicialmente a grupos de niños del primer grado en colegios públicos urbanos y privados de las ciudades de Puebla, Tehuacán y San Pablo del Monte. La aplicación comenzó como parte de las tesis del posgrado, luego, la apertura del colegio Kepler dio la oportunidad de aplicar el método de forma sistemática y organizada, como una metodología institucional.

El método se ha dado durante 6 años consecutivos en el primer grado de educación primaria y ha permitido realizar algunas modificaciones y adaptaciones que se presentan en un libro (Solovieva y Quintanar, 2014); todo el diseño y la experiencia se basan en el enfoque del paradigma histórico-cultural (Solovieva, 2014) y la teoría de la actividad aplicada a la enseñanza (Talizina, 2009; Talizina, Solovieva y Quintanar, 2010).

\section{Paradigma histórico-cultural}

En la psicología y pedagogía contemporáneas hay una aproximación cuyo principio esencial es que todo desarrollo psicológico del niño es histórico-cultural, que surge de la adquisición de la cultura, es decir, de la experiencia humana que se organiza, conserva y produce con medios culturales: fuera del organismo (Vigotsky, 1996; Lotman, 2001). Se considera que el niño no adquiere dicha experiencia por causas biológicas o sociales amplias, sino por medio de la interacción cultural constante en la sociedad y junto a otros. Así, el desarrollo intelectual y la formación de conceptos no se dan de forma natural ni dependen de una interacción social poco definida, sino del tipo preciso de instrucción que recibe el niño en la escuela (Vigotsky, 1992).

A esta interacción se le puede concebir como una forma de diálogo constante en la cual el niño se introduce a partir de su nacimiento, y que le prepara para tomar conciencia de su propia conducta por medio de la reflexión sobre el proceder de los demás (Bajtín, 2000). Dicha reflexión no es automática, sino que puede y debe ser formada en un proceso de actividad organizada; con ello, la enseñanza escolar puede cambiar radicalmente la

3 Es posible consultar en www.neuropsicología.buap.mx

4 Es posible consultar en www.colegiokepler.edu.mx 
cualidad de conceptos y conocimientos del niño, garantizando su desarrollo psicológico y la transformación de la actividad escolar (Leontiev, 1981); esto se relaciona necesariamente con el interés cognitivo del niño y con la consolidación de los motivos de su personalidad (Leontiev, 1983).

La actividad escolar, como nueva forma de acción en la vida del niño, implica ciertas características del desarrollo y de la personalidad, como la voluntad, la motivación cognoscitiva, la imaginación y la posibilidad de usar signos y símbolos como medios propios (Salmina, 1985; Salmina y Filimonova, 2001, 2002; Elkonin, 1989, 1996; Obukhova, 1996; Davidov, 1996). La organización del proceso de enseñanza de lectura y escritura requiere que el profesor tome en cuenta las nuevas formaciones del niño, producto de las nuevas influencias originadas en el acto escolar y de comprender el plano simbólico en el que transcurre el lenguaje escrito.

La formación de la actividad escolar depende de que el alumno tome conciencia de las regularidades de asimilación del proceso (Talizina, 1998), y para que reflexione sobre el objeto de su conocimiento, dicha idea debe ser formada en sus maestros. Al respecto, Talizina (2000) afirma que asimilar cualquier proceso implica acciones cognoscitivas de los escolares; no existe un conocimiento puro, si alguien quiere aprender algo, necesariamente aprenderá a hacer algo con estos saberes (Eco, 2005). La enseñanza se puede concebir como formación de un sistema de acciones que el niño aprenderá a realizar con ayuda del pedagogo. El método es eficaz siempre que considere todo el sistema de acciones incluidas en sus diversas etapas.

\section{Método innovador para la introducción en la lectura}

Algunos (Pérez, 2004) han señalado la inutilidad de acercarse tradicionalmente a la lectura, enfatizando en la necesidad de reconsiderar los programas de enseñanza y los métodos pedagógicos en la escuela primaria; como alternativa, surge el método original para la introducción inicial de la lectura, basado en el análisis fonético-fonemático del idioma y en la construcción del esquema materializado de la palabra (Elkonin, 1989).

Sin embargo, a pesar de que las teorías de Vigotsky y de Luria son populares, llegando a incluirse en los planes de estudios de psicología de diversos países de América Latina, como otras prácticas, este método es desconocido para muchos especialistas. Frecuentemente, las teorías llegan a los lectores con interpretaciones específicas, acentuando la colaboración social conjunta de los niños para la solución de diversos problemas. Como ejemplos se pueden señalar los conceptos de la "zona del desarrollo próximo" y del "origen social de la psique humana" (Rogoff, 1993). En algunos casos, dicha interpretación simplemente cambia por completo el sentido de la teoría por una combinación con los conceptos de la teoría cognitiva y constructivista (Feuerstein, et al., 1980).

Así, se supone que para formar los hábitos intelectuales (lectura) en niños de edad escolar inicial, con problemas en el aprendizaje, es suficiente con garantizar su actividad conjunta de juego o de lectura, en la que los alumnos capaces “ayudan” a los débiles (Cole, 1997). Se hace énfasis en los contextos sociales colaborativos sin precisar los objetivos exactos en las tareas, los niveles de introducción, la edad psicológica o los contenidos de las áreas de conocimiento.

Como prueba de lo anterior, los autores del proyecto "Quinta Dimensión”, coordinado desde la Universidad de san Diego, California, proponen un contexto social amplio como escenario para el desarrollo, desde, por ejemplo, ejercicios en los que, durante la realización del análisis fonético de las palabras del idioma inglés, se plantea al alumno un juego computacional en el cual gana quien adivina la pronunciación correcta, reforzando la adivinanza mientras que la "ayuda" del adulto o de un compañero mayor consiste en que éste pronuncia correctamente las palabras.

Viendo lo anterior, surgen entonces interrogantes: ¿cuál es la diferencia entre este método de enseñanza y corrección, también planteada por el autor, y la presentación de la "ayuda", de los métodos clásicos de enseñanza propuestos por los conductistas (Skinner, 1938), utilizados constantemente por pedagogos de todo el mundo?; ¿por qué el autor cita a los representantes de la teoría histórico-cultural?

La difusión de la teoría de la enseñanza debe partir de fuentes directas, de los representantes y seguidores de ésta (Talizina, $2000,2001)$. La elaboración y uso de métodos de enseñanza basados en la teoría de la actividad en otros países, puede no solo ayudar a comprender mejor la esencia psicológica de la naturaleza histórico-cultural de la psique humana, sino a solucionar muchos problemas prácticos de la educación en todos los niveles, tanto normal, como en los casos que requieren de una atención diferenciada por motivo de discapacidades o lesiones de nacimiento (Quintanar y Solovieva, 2000; Manske, 2004).

En el caso concreto de la formación del hábito inicial de lectura, la aplicación de la teoría de la actividad de enseñanza en la práctica pedagógica requiere de un método concreto, que responda a los principios de organización fonético-fonemática del idioma correspondiente (que se va a enseñar). La lectura se concibe como una de las formas esenciales de la actividad verbal 
y cumple con funciones sociales, comunicativas, intelectuales, cognoscitivas y regulativas, además de apoyar la construcción del reflejo mediatizado de la realidad.

Así, de acuerdo con Luria (1997), el proceso de lectura y escritura no trata solo de la decodificación, sino de la transformación de símbolos gráficos visuales en un sistema del lenguaje oral a través de la articulación; además, el niño debe emplear el mismo código que ve y representarlo de forma escrita. Durante la formación, pasa por diferentes etapas hasta alcanzar su automatización e interiorización. La organización se da a partir del método de las acciones mentales por etapas de Galperin $(1976,2000)$ y Talizina $(1984,2000)$, de acuerdo al cual todas las acciones intelectuales pasan por las etapas material y materializada, perceptiva, y verbal externa e interna.

En cada etapa del trabajo el maestro debe garantizar la motivación de los niños, el deseo y la disposición. Las ayudas se pueden dar de diferentes maneras: la animación, las preguntas del profesor o mostrar ejemplos de ejecución de tareas similares; se debe recordar que las ayudas dependen de la zona de desarrollo próximo del niño: entre más pequeña sea la ayuda, más grande será su zona de desarrollo próximo y a la inversa (Solovieva, 2014).

\section{Etapas del método}

Es importante anotar que el lector se puede acercar al texto Enseñanza de la lectura: Método práctico para la formación lectora (Solovieva y Quintanar, 2014) para conocer con detalle todo el proceso de aplicación; por ahora solo es posible exponer en forma resumida las etapas esenciales del método formativo:

1. Análisis fonético oral de las palabras del idioma español:

- Identificación de sonidos acentuados y no acentuados.

2. Análisis fonético con apoyo en la acción externa:

- Introducción del esquema materializado de sonidos de la palabra.

- Materialización de sonidos vocales.

- Materialización de sonidos vocales acentuados.

- Materialización de sonidos consonantes.

- Materialización del sonido blando "N"N" y duro "N".

- Materialización del sonido largo "RR" y corto "R".

3. Análisis fonético con el apoyo en la acción gráfica:

- Representación gráfica del esquema de sonidos de la palabra.

- Representación gráfica de sonidos vocales.

- Representación gráfica de sonidos consonantes.

- Representación gráfica de la suavidad del sonido "Ñ".

- Representación gráfica de la longitud del sonido "RR".
4. Introducción de letras con el apoyo en la acción materializada:

- Introducción de letras que corresponden a los sonidos vocales.

- Introducción de letras que corresponden a los sonidos consonantes (en orden de complicación de la relación sonido-letra que existe en el idioma español).

5. Introducción de la acción de escritura de letras:

- Escritura de letras que determinan vocales.

- Escritura de letras que determinan consonantes.

6. Lectura y escritura independiente de palabras.

7. Análisis fonético oral de las palabras.

8. Tareas diversas de lectura y escritura en grupos, con el paso a la actividad individual.

Durante esta etapa solo se trabaja con las palabras en el nivel del lenguaje oral; el maestro pronuncia y los niños escuchan, después los niños pronuncian palabras frecuentes y novedosas. Durante la presentación el maestro acentúa cada uno de los sonidos de la palabra y pronuncia las palabras con diferente acentuación.

\section{Análisis fonético materializado}

Esta etapa implica la materialización de la palabra pronunciada con ayuda de esquemas externos. Con la colaboración del maestro, los alumnos dibujan los esquemas para las palabras y los recortan con tijeras; los esquemas son celdas que corresponden a la cantidad de sonidos de las palabras (se trata de la cantidad de sonidos y no de letras), por ejemplo, para la palabra "hombre" hay 5 sonidos (a pesar de que tiene 6 letras).

\section{La representación materializada de los sonidos vocales y consonantes}

En esta etapa del trabajo formativo el maestro debe explicar a los niños que es necesario aprender a distinguir entre vocales y consonantes, expresándoles que para señalar las vocales en el esquema utilizarán círculos rojos, identificando el lugar de las vocales en la palabra y sustituyendo los círculos blancos por rojos. Una actividad que puede reforzar esta acción se puede hacer desde el juego, por ejemplo, el niño puede buscar palabras que correspondan al esquema dado.

\section{Representación materializada del acento, sonidos largos y cortos, sonidos blando y duro}

Durante esta fase el maestro debe explicar a los alumnos que en nuestro idioma las palabras tienen diferente pronunciación, algunas son más fuertes que otras, y que aquellas con sonidos más 
fuertes se llaman acentuadas. Al tiempo, se deben dar algunos ejemplos de cambios de acentuación prosódica en las palabras, que se puede realizar voluntariamente, realizar diferenciaciones entre el sonido largo "rr" y el corto " $r$ ", y entre el sonido blando "ñ" y duro "n", y plantear y discutir diversos ejemplos del uso de dichos sonidos en las palabras.

\section{Introducción de letras por orden, desde la relación única entre fonema y grafema, hasta los tipos de relaciones complejas entre el nivel de los sonidos y el nivel de las grafías}

La introducción de letras inicia con aquellas que determinan los sonidos vocales, lo cual se relaciona con la situación de correspondencia recíproca de sonido-letra y entendiendo que los sonidos vocales se identifican con mayor claridad durante el análisis fonético de las palabras. Inicialmente la introducción de las letras se trabaja en el plano de esquemas de palabras que los niños ya conocen, para luego comenzar con la parte más compleja del proceso de formación de lectura: la introducción de letras que corresponden a los sonidos consonantes, que también inicia con los casos de correspondencia única entre el sonido y la letra que lo representa.

En la siguiente etapa se introducen las letras que pueden representar dos sonidos diversos: [G] J] y [S] [K]. La orientación se dirige hacia el sonido siguiente, de lo cual precisamente depende la elección de la letra. Es decir, se acostumbra al niño escuchar con atención todos los sonidos dentro de las palabras, determinando el primero, segundo, etc., y, en caso de elegir entre la letra "g" o "j”, tiene que determinar la vocal que sigue al sonido consonante. Veamos un ejemplo de la regla para la orientación hacia la vocal siguiente dentro de la palabra:

1) $g+o, u, a=[G]$

2) $g+e, i=[J]$

Las reglas se explican y se elaboran junto a los niños en el pizarrón y en sus cuadernos, para que permanezcan a la vista durante el análisis de diversos ejemplos en las sesiones escolares. Las palabras que corresponden a estos ejemplos pueden ser: "domingo"; "giro" o "gelatina".

Los casos de relaciones más complejas entre sonido y letra (v, b, z, s, c) rebasan las posibilidades de la etapa preescolar, debido a que éstas se deben apoyar en las relaciones léxicas y morfológicas más amplias del idioma español, lo cual es imposible sin dominar el hábito inicial de la lectura. Así, en esta etapa, los objetivos de la formación se han alcanzado; para trabajar con las letras correspondientes, en cada ocasión el maestro indica al niño con qué letra se escribe (o construye) la palabra correspondiente.

\section{Lectura independiente de palabras, oraciones y textos}

En esta fase los niños inician la lectura de las palabras de manera independiente, utilizando en todas las ocasiones el esquema gráfico para representar la palabra correspondiente; gradualmente, se retiran los esquemas y se pasa a la lectura de oraciones y textos.

\section{Aportes}

Como resultado de la aplicación de este método es posible destacar que se ha presentado un aumento en el nivel de reflexión sobre las propias acciones de lectura; los niños logran ejercer exitosamente la lectura independiente de palabras y oraciones sencillas, además de la escritura inicial. Al tiempo, se garantiza una toma de conciencia sobre la palabra como objeto específico de análisis y asimilación, lo cual, de acuerdo a la opinión de muchos psicólogos, es una condición indispensable para la formación de conceptos lingüísticos (Leontiev, 1981; Karpova, 1981; Talizina, 2000).

Lo anterior es muy importante, pues solo la formación sobre la palabra como objeto específico de análisis puede garantizar una visión teórica del mundo, y ésta debe ser alcanzada a través del proceso de enseñanza-aprendizaje escolar. Los conceptos no surgen de la experiencia inmediata del niño, ni son garantizados por los procesos madurativos fisiológicos, como lo consideran algunos autores (Piaget, 1954). La organización adecuada de la adquisición histórico-cultural humana, que constituye la fuente del desarrollo psicológico del niño, es la fuente del nivel de formación en estos conceptos (Galperin, 2000).

El método contribuye a la educación al usar una base invariable de la codificación y decodificación, mediada por signos lingüísticos, junto a la comprensión reflexiva de los niños, que se suman a la inclusión de una base orientadora de la acción para realizar actividades gráficas en la etapa preescolar, y al uso del análisis y la síntesis espacial en los juegos, útil como prevención de problemas en el aprendizaje. La ejecución gradual de las tareas y la organización de juegos, combinadas con la orientación y la verificación, inicialmente por parte del adulto, y luego del mismo niño, ayudan a la formación del control de la actividad general, 
uno de los logros principales de la edad preescolar (Salmina y Filimonova, 2001; Obukhova, 1996; Elkonin, 1996, 1999).

Este método de formación de lectura incluye una ordenación espacial en los niveles materializado y perceptivo, y una regulación constante (del adulto hacia la niño) de la ejecución de todas las tareas. Así, tiene ventajas en comparación con los métodos tradicionales. En primer lugar, se apoya en el análisis fonético-fonemático del idioma materno, necesario para decodificar el lenguaje escrito, el cual se debe formar como acto consciente del niño para pasar al nivel automatizado, garantizando los objetivos conscientes de orden superior, como asimilar el sentido profundo de la obra literaria o analizar el estilo artístico; alcanzar dichos objetivos es imposible sin adquirir antes el hábito de lectura interna. La escuela tradicional no cumple con ello y eso se manifiesta en el fracaso escolar generalizado y en la pobreza de hábitos lectores.

En segundo lugar, emplear la construcción del esquema externo de la palabra, en el plano sonoro y materializado, permite al niño adquirir la habilidad para sentir el inicio y el final de la palabra, es decir, para formarse una imagen global de ella. Esto lleva a la posibilidad de utilizar la organización espacial de la palabra en la escritura propia del niño y de conservar adecuadamente los espacios entre palabras. Como se sabe, la unión y separación inadecuada de palabras en la escritura es uno de los problemas que no encuentran solución dentro de la pedagogía tradicional.

En tercer lugar, el presente método ofrece la ventaja de que el niño no debe pasar de la lectura por silabeo a la lectura automatizada de palabras, debido a que durante el análisis de sonidos pronuncia, desde el inicio, la palabra completa, a partir de la cual identifica los sonidos. En cuarto lugar, desde comienzo se separan los conceptos "sonido" y "letra", hecho que permite anticipar dificultades posteriores en el aprendizaje y evitar la confusión para distinguir sonidos y letras, que lleva a los errores ortográficos, de anticipación y a la lectura adivinatoria, que llegan hasta niveles medios y superiores de enseñanza, problemas que ni el método global ni el analítico, o cualquier combinación empírica, logran solucionar.

\section{Consideraciones importantes}

El sistema de relaciones entre profesores y alumnos permite al maestro desarrollar acciones con significado social, para así crear las condiciones de asimilación de la actividad escolar (Talizina,
2000). El objeto de las acciones puede ser material, externo o perceptivo, pero también una palabra o una idea. De hecho, la esencia de la enseñanza es que debe conducir al niño a la posibilidad de operar, no solo objetos reales, sino generalizados, abstractos y sistematizados en materias de saberes específicos (Davidov, 1996, 2000). A diferencia de la experiencia empírica, la enseñanza debe llevar al niño a la experiencia cultural teórica; la introducción a la lectura es el paso necesario para llegar a la cultura general humana.

Se debe subrayar que la formación de cualquier proceso escolar con orientación activa considera la acción del sujeto que la realiza y quien, naturalmente, siempre manifiesta su individualidad en ella. Una acción cognitiva implica la presencia de un motivo cognitivo y un objetivo consciente y preciso de su realización. En la práctica tradicional de la escuela no se establecen los motivos cognitivos, ni se piensa en objetivos claros para los niños; frecuentemente los maestros no entienden las metas de la enseñanza, por lo que la escuela tradicional no contribuye al proceso de formación de habilidades o de formación de la personalidad, incumpliendo con su papel formador, no logra introducir el proceso lector de forma eficaz.

Dichos objetivos tampoco se alcanzan con las propuestas basadas en la teoría constructivista, cuando se pretende observar cómo los niños, por su propia cuenta, logran desarrollar procesos de aprendizaje desde una interacción social. Así, los estudios efectuados por Ferreiro y Gómez (1982) en niños pequeños sin experiencia curricular, ponen en evidencia sus procesos de asimilación; al niño se le pide que escriba o que lea algo sin que sepa leer o escribir, y pareciera que es un sujeto libre de las influencias sociales, al cual solo después de haber creado las competencias de asimilación le es posible acceder a los demás sujetos y a su influencia. Una vez más se encuentra la idea de que no hay otros, solo hay un sujeto.

Así, no solo no se toma en cuenta el papel del adulto ni la orientación específica propia del contenido de la formación del proceso de lectoescritura, sino que tampoco se considera la posibilidad de una formación de la capacidad de asimilación, la cual, en este estudio, se llama procedimientos lógicos del pensamiento, los cuales impactan en la consolidación de la lectoescritura. Los métodos concretos que se plantean son una variante del método global, basado en la asociación visual de la imagen global de una palabra y en la adivinanza, por parte del niño, de su significado en las oraciones a la hora de escuchar la lectura que realiza el pedagogo. 
Lo anterior sugiere que el proceso de lectoescritura se debe dar como competencia intrínseca del sujeto, y dichas ideas han sido retomadas en diversas publicaciones (Fons Esteve, 2013). Se puede pensar que se trata de enseñar a partir de la experiencia del niño, sin contar con la necesidad de una preparación previa o de considerar el contenido de la actividad particular y los tipos de orientación; un punto de vista bien conocido y ampliamente difundido entre los maestros, relacionado con el enfoque actual por competencias (Carretero, 2002; Cázares, 2012; Argudín, 2012).

Como es bien sabido, no hay un programa concreto de formación de procedimientos lógicos del pensamiento que deba ser desarrollado durante una asignatura; así, el procedimiento de formación se hace en términos muy generales, sin recurrir a los contenidos indispensables, su mecánica de funcionamiento y los cursos. Esto lleva a que las actividades cognoscitivas se formen de manera circunstancial y a que, por ende, el alumno se vea imposibilitado para manejar adecuadamente las acciones que garantizan la asimilación de un procedimiento lógico.

A diferencia de la enseñanza tradicional y del enfoque constructivista, este método innovador considera el contenido de la acción de la lectura a partir de un análisis de los sonidos como elementos de las palabras. Se meditan los niveles de introducción de la acción desde el plano simbólico externo (Solovieva y Quintanar, 2014) y se llega a un trabajo guiado por el maestro y siempre colectivo en las sesiones.

En la actualidad este método se utiliza de forma constante y planeada en el Colegio Kepler de la ciudad de Puebla. Existe una variante modificada y organizada para la introducción de la lectu- ra en el idioma inglés, que se trabaja a partir del segundo grado de primaria, cuando los niños ya utilizan de forma consolidada este proceso con el idioma español (Solovieva, 2015). Los maestros de escuela primaria conocen y manejan el método de manera reflexiva y consciente, aportando modificaciones y ejemplos novedosos durante el proceso educativo.

Adicionalmente, se ha desarrollado una aplicación del método para Tablet (iPad), denominada Leer Bien, que se recomienda utilizar como apoyo complementario en casa. Es posible encontrar la aplicación en la Apple Store y, muy pronto, también estará disponible en otros sistemas. ¡Invitamos a los lectores a que conozcan nuestra propuesta!

Imagen 1. Leer bien. Aplicación para iPad

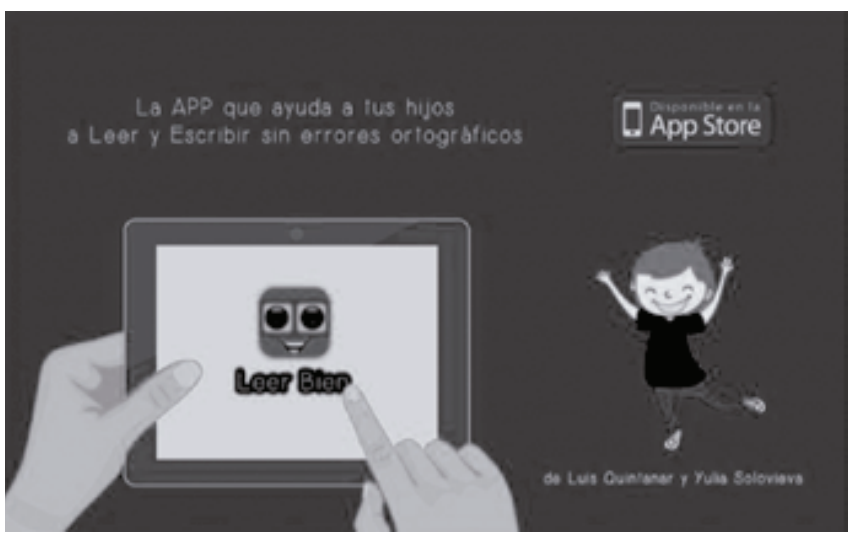

\section{Referencias}

Argudín, Y. (2012). Educación basada en competencias. México: Trillas.

Bajtín, M. (2000). Autor y su héroe. Hacia las bases filosóficas de las ciencias humanitarias. Moscú: Academia.

Carretero, M. (2002). Construir y enseñar. Madrid: Visor.

Cázares, L. (2012). Estrategias educativas para fomentar competencias. México: Trillas.

Cole, M. (1997). Cultural psychology. A once and future Discipline. Cambridge: Cambridge University Press.
Davidov, V. V. (1996). La teoría de la enseñanza que conduce al desarrollo. Moscú: INTER.

Davidov, V. V. (2000). Tipos de generalización en la enseñanza. Moscú: Sociedad Pedagógica de Rusia.

Eco, U. (2005). Tratado de semiótica general. México: De Bolsillo.

Elkonin, D. B. (1989). Obras psicológicas escogidas. Moscú: Pedagogía.

Elkonin, D. B. (1996). Desarrollo psicológico en las edades infantiles. Moscú: Academia de Ciencias Pedagógicas y Sociales. 
Elkonin, D. B. (1999). Psicología del juego. Moscú: Vlados.

Ferreiro, E., y Gómez, P. M. (1982). Nuevas perspectivas sobre los procesos de lectura y escritura. México: Siglo XXI.

Feuerstein, R., Rand, Y., Hoffman, M., y Miller, R. (1980). Instrumental enrichment: An intervention program for cognitive modification. Baltimore: Baltimore University Park Press.

Fons Esteve, M. (2013). Leer y escribir para vivir. México: Biblioteca Infantil.

Galperin, P. Y. (1976). Introducción a la psicología general. Moscú: Universidad Estatal de Moscú.

Galperin, P. Y. (2000). Cuatro conferencias sobre psicología. Moscú: Escuela Superior.

Karpova, S. N. (1981). El desarrollo de la orientación hacía el lenguaje y sus elementos. En I. Iliasov y V. Liaudis. (Eds.), Compilación sobre psicología del desarrollo y psicología pedagógica (pp. 220-226). Vol. 2. Moscú: Universidad Estatal de Moscú.

Leontiev, A. A. (1981). Concientización del significado por el portador del idioma. En En I. Iliasov y V. Liaudis. (Eds.), Compilación sobre la psicología del desarrollo y psicología pedagógica. (pp. 227-231). Vol. 2. Moscú: Universidad Estatal de Moscú.

Leontiev, A. N. (1983). Obras escogidas. Moscú: Universidad Estatal de Moscú.

Lotman, Y. M. (2001). Semio-esfera. San Petersburgo: Arte-San Petersburgo.

Luria, A. R. (1997). Conciencia y lenguaje. Madrid: Visor.

Manske, Ch. (2004). Entwicklungsorientierter Lese und Scbreibunterricht fur alle Zinder. Die nichtlineare Didaktik nach Vygotskij. Beltz, Verlag: Sonderpadogogik.

Obukhova, L. F. (1996). Psicología del desarrollo por edades. Moscú: Agencia Pedagógica de Rusia.

Olmos, A., Morales, I., y Álvarez, S. (2002). Una propuesta de cultura colaborativa: ideas fundamentales en la educación universitaria. En A. Olmos (Ed.), Aplicaciones educativas de la teoría sociocultural (pp. 2-38). México: Facultad de Estudios Superiores Zaragoza.

Pérez, M. R. (2004). Sociedad del conocimiento y refundación de la escuela desde el aula. Lima: Libro Amigo.

Piaget, J. (1954). The construction of reality in the child. New York: Basic Books.
Quintanar, L., y Solovieva, Y. (2000). La discapacidad infantil desde la perspectiva neuropsicológica. En M. A. Cubillo, J. Guevara y A. Pedroza (Eds.), Discapacidad humana, presente y futuro. El reto de la rehabilitación en México (pp. 51-63). Tlaxcala: Universidad del Valle de Tlaxcala.

Rogoff, B. (1993). Aprendices del pensamiento. El desarrollo cognitivo en el contexto social. Barcelona: Paidós.

Salmina, N. G. (1988). El signo y el símbolo en la enseñanza. Moscú: Universidad Estatal de Moscú.

Salmina, N. G., y Filimonova, O. G. (2001). Diagnóstico y corrección de la actividad voluntaria en niños preescolares y escolares. México: Universidad Autónoma de Tlaxcala.

Salmina, N. G. y Filimonova, O. G. (2002). Problemas de aprendizaje de las matemáticas básicas y su corrección. Puebla: Instituto Universitario de Estudios Avanzados.

Skinner, B. F. (1938). The behaviour of organisms. New York: Appleton Century Crofts.

Solovieva, Y. (2014). La actividad intelectual en el paradigma bistórico-cultural. México: CEIDE.

Solovieva, Y. (2014). Estrategias introductorias del lenguaje escrito en el idioma inglés. México: Plaza y Valdés.

Solovieva, Y., y Quintanar, L. (2014). Enseñanza de lectura. Método práctico para la formación lectora. México: Trillas.

Talizina, N. F. (1984). Dirección del proceso de asimilación de conocimientos. Moscú: Universidad Estatal de Moscú.

Talizina, N. F. (1998). La educación hoy. México: Instituto de investigaciones de problemas de aprendizaje.

Talizina, N. F. (2000). Psicología pedagógica. San Luis Potosí: Universidad Autónoma de San Luis Potosí.

Talizina, N. F. (2001). La formación de las habilidades matemáticas en niños escolares. San Luis Potosí: Universidad Autónoma de San Luis Potosí.

Talizina, N. F. (2009). Aplicación de la teoría de la actividad a la enseñanza. México: Universidad Autónoma de Puebla.

Talizina, N., Solovieva, Y., y Quintanar, L. (2010). La aproximación de la actividad en psicología y su relación con el enfoque histórico-cultural de L.S. Vigotsky. Novedades educativas, No. 22, 230, pp. 4-9.

Vigotsky, L. S. (1992). Obras escogidas. Tomo II. Madrid: Visor.

Vigotsky, L. S. (1996). Obras escogidas. Tomo IV. Madrid: Visor. 